\title{
Optic Nerve Cavernous Haemangioma as a Rare Cause of Retro-Orbital Pain mimicking Intracranial Aneurysm.
}

\section{Doukas A*, Ahmeti H, Barth H, Mehdorn M and Nabavi A}

Department of Neurosurgery, University Clinics Schleswig-Holstein, Kiel, Germany

${ }^{*}$ Corresponding author: Doukas A, Department of Neurosurgery, University Clinics Schleswig-Holstein, Arnold- Heller Str. 3, Haus 41, 24105 Kiel, Germany, E-mail: alexdouk@hotmail.de

Citation: Doukas A, Ahmeti H, Barth H, Mehdorn M, Nabavi A (2014) Optic Nerve Cavernous Haemangioma as a Rare Cause of Retro-Orbital Pain Mimicking Intracranial Aneurysm. J Case Rep Stud 2(5): 504. doi: $10.15744 / 2348-9820.2 .204$

Received Date: August 10, 2014 Accepted Date: October 07, 2014 Published Date: October 10, 2014

\begin{abstract}
Cavernous haemangiomas of the optic nerve, optic chiasm or optic tract are rare. Usually they present with acute onset of symptoms such as acute decline of visual acuity, headaches, nausea or even decline of the level of consciousness which suggests haemorrhage in or even out of the lesion. Otherwise, they have an insidious clinical pattern with subacute or chronic visual disturbance, diplopia and retro- orbital pain.

We present a case of a 42 -year-old male patient with pain behind the left bulbus and progressive unilateral visual loss and hemianopsia who was admitted to our clinic with the possible diagnosis of an aneurysm of the left internal carotid artery. The diagnostic work up included thin layer MRI, digital angiography and CT wherein the assumed diagnosis was cavernous haemangioma or tumor of the optic nerve. Total excision of the lesion was achieved via a left frontolateral approach resulting in marked improvement of the visual acuity and the visual field. The histological evaluation confirmed the diagnosis of a cavernous haemangioma.
\end{abstract}

Keywords: Cavernous haemangioma; Optic nerve; Aneurysm; Frontolateral approach

\section{Introduction}

Eye pain, periorbital and retro-orbital pain are common symptoms, with which many patients present to their ophthalmologist or neurologist. There are numerous causes of such symptoms, which sometimes require immediate or at least prompt therapy. Cerebral cavernous malformations are low-flow vascular lesions, which are typically angiographically occult, have an incident of $0.3 / 0.7 \%$ in the general population and account for $10-20 \%$ of all vascular malformations [1]. Most of them occur supratentorially, $15 \%$ of them are found infratentorially. $5 \%$ of the cavernomas are found in the spinal cord extra- or intradurally [2]. Cavernous haemangiomas of the anterior optic pathway are very rare [3] and either present with acute, subacute or chronic symptoms. They are occult vascular malformations which can also have hereditary or sporadic distribution [4].

We present a rare case of cavernous haemangioma of the optic nerve which mimicked an aneurysm of the internal carotid artery or even a tumor, displacing the optic nerve and optic chiasm and causing retro-orbital pain. After total removal of the lesion via a left frontolateral approach, the visual acuity as well as the visual field deficit rapidly improved. The pain disappeared.

\section{Case presentation}

A 42-year-old male patient presented to his general practitioner complaining about progressive decline of his visual acuity on the left side and retro-orbital pain. He was further evaluated from an ophthalmologist who diagnosed left upper and lower quadrant visual field deficit. Under the suspicion of a space occupying lesion pressing the optical nerve or chiasm, an MRI was performed. Herein a thrombosed aneurysm of the internal carotid artery was diagnosed and the patient referred to us for further evaluation and treatment. There were no abnormalities of the ocular movement or other neurological deficits noted.

\section{Imaging}

The first diagnostic modality used was an MRI, since a sellar lesion was initially suspected. The results where however inconclusive and because of the configuration of the lesion in the T2 and T1 sequence with contrast medium an aneurysm of the left internal carotid artery was suspected (Figure 1A-E). Thus, a digital subtraction angiography was performed. Neither aneurysm nor arteriovenous malformations could be seen. A CT scan was additionally performed to rule out calcifications. Hence, two hypothetical differential diagnoses were considered; a cavernous haemangioma or a tumor with intralesional hemorhage of the optic nerve. 


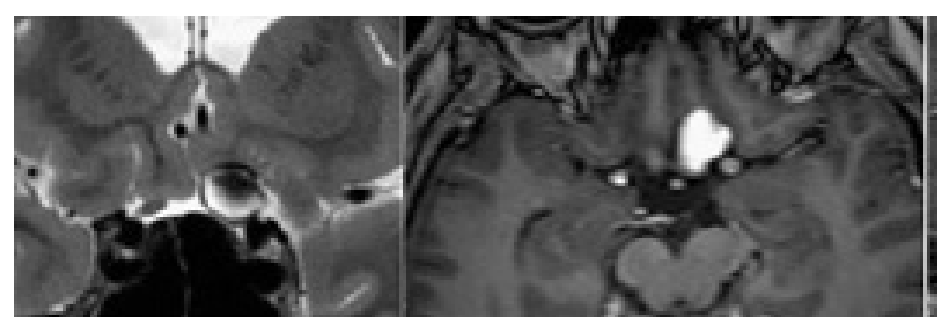

A

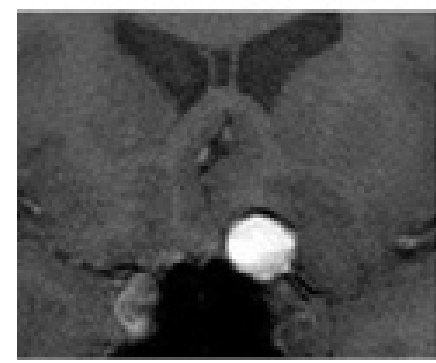

D

B

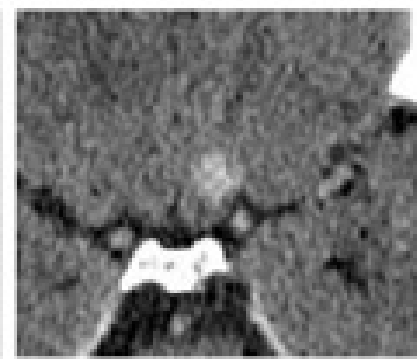

$\mathrm{E}$

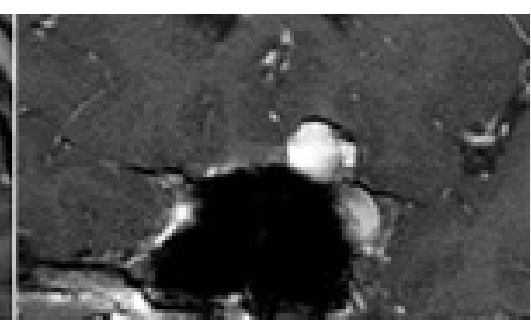

c

Figure 1: A. Coronal T2- weighted MRI showing optic nerve being flattened medially and downwards, various fluid intensities B. Axial T1-weighted MRI with contrast medium showing enhancement

C. Coronal T1-weighted MRI with contrast medium showing the proximity of the lesion to the internal carotid artery

D. Coronal T1-weighted MRI without contrast medium

E. Axial CT scan showing hyperdensity of the lesion

\section{Operation}

A left frontolateral approach was selected. The optic nerve was exposed and after dissecting the falciform ligament the entire lesion was exposed. Hemosiderin desposits were not limited to the lesion, but extended to the frontal gyrus suggesting prior repeated hemorrhage. The lesion had the typical appearance of a cavernoma, showing multilobulated, mulberry-like bulging (Figure 2A). A thin layer of overlying Glia was entered, the accompanying hematoma removed. Using a micronerve hook, the cavernoma could be removed from the optic nerve (Figure 2B). The surrounding Optic nerve displayed multiple shaedes of xanthochromia, supporting our impression of prior bleeding episodes. These areas were left untouched.
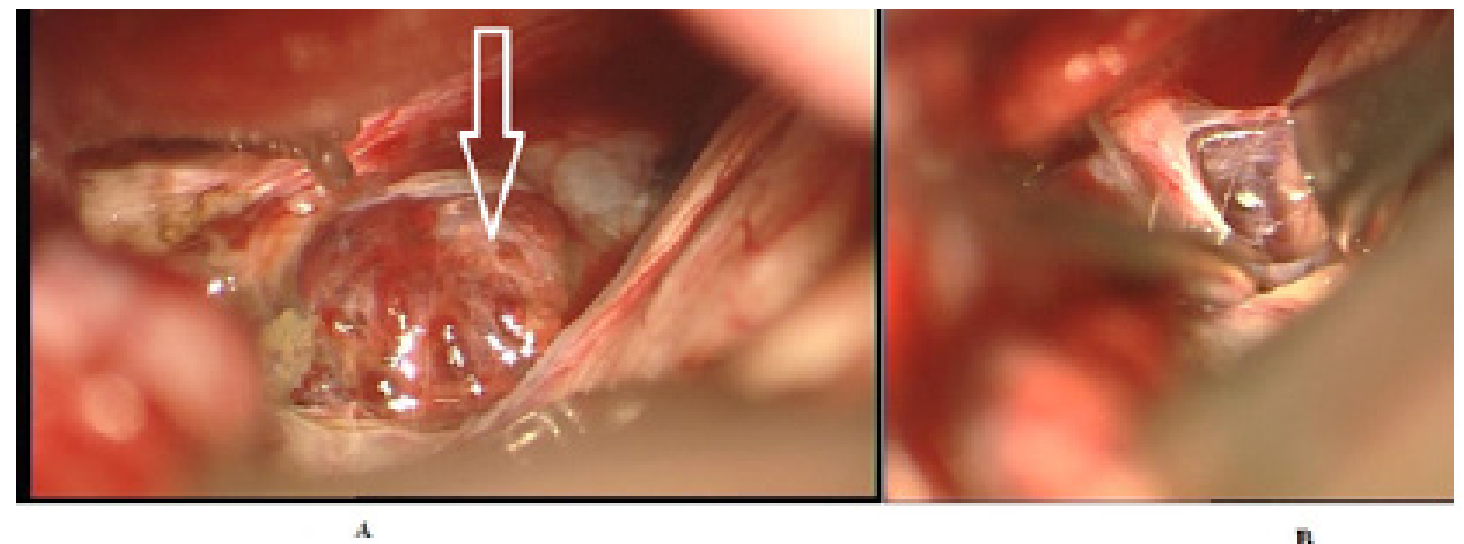

Figure 2: A. Mulberry-like appearance, that extends superiorly from the optic nerve, pushing the optic nerve apart like a wedge (arrow)

B. Intraoperative photo after complete resection of the cavernoma. The prior location of the cavernoma is clearly seen, the nerve flattened and broadened by the initial mass

The postoperative course was uneventful. The MRI confirmed the total removal of the tumor without complications (Figure 3A-C).

The visual acuity of the left eye increased from 0.6 to 0.8 only 2 days after the operation. Moreover, a marked improvement of the visual field deficit could be assessed (Figure 4A-D). The retro-orbital pain disappeared. 


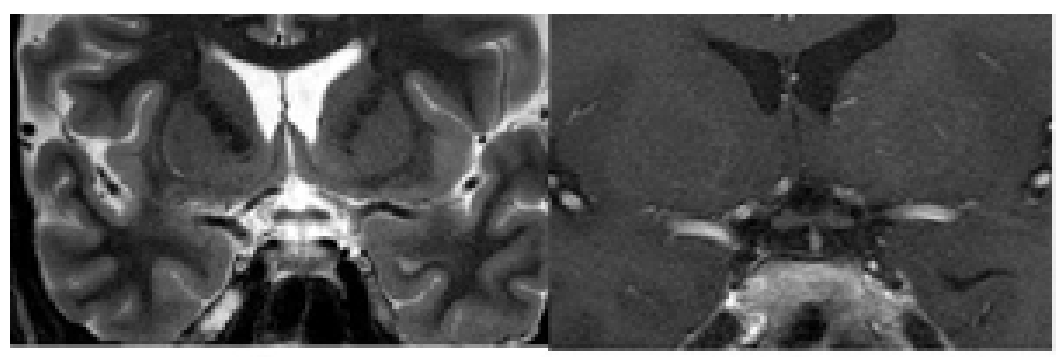

A

C

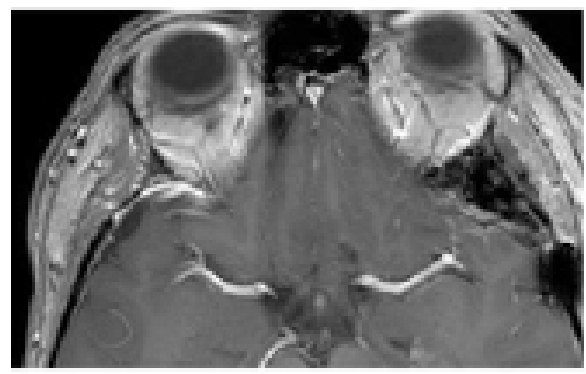

B

Figure 3: Post-operative MRI confirming the total removal of the lesion

A. Coronal T2-weighted MRI with fully recovered normal anatomy of the nerve and chiasm

B. Axial T1-weighted MRI post- contrast without residual tumor. On close scrutiny, the previous location of the mass can be still discerned

C. Coronal T1-weighted MRI post-contrast
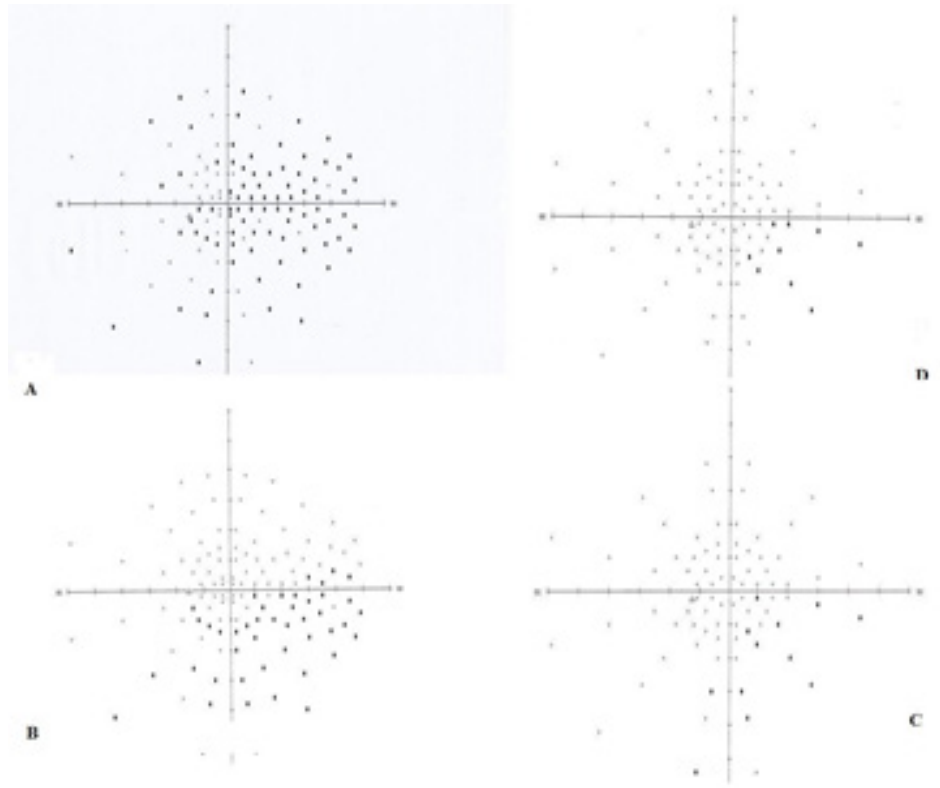

Figure 4: A. Visual field deficit of the left eye preoperatively

B. Visual field 2 days after the operation with obvious improvement

C. Visual field 3 weeks after the operation 5 weeks after the operation the visual field is almost back to norm

D. 5 weeks after the operation the visual field is almost back to normal

\section{Discussion}

There are many causes of periorbital and retro-orbital pain which are mostly treated by ophthalmologists but also by neurologists. That is, because not only eye but also intracranial pathologies affecting the optic nerve, can cause pain in the "quiet" eye. Since the pain is usually combined with headaches and visual deficits, the patients are highly concerned and fear the worst diagnosis such as tumors or even brain hemorrhage [5]. 
The most common etiology includes ocular processes such as open-angle glaucoma, angle-closure glaucoma, corneal disease, uveitis, scleritis and episcleritism intraocular tumors, ocular ischemia, or suprachoroidal hemorrhage. There are also trauma causes being described such as sclera laceration or rupture. Lastly, retina or choroid processes could also provoke such symptoms.

Another category of processes which lead to periorbital pain are those affecting the optic nerve. These are optic neuritis, neuroretinitis, ischemic optic neuropathy or compressive optic neuropathy [6]. The last group is of neurosurgical interest, since they encompass a variety of tumors affecting the optic pathways such as meningiomas, gliomas, craniopharyngiomas, pituitary adenomas, lymphomas, germinomas, nasopharyngeal cancer with intracranial expansion or metastatic tumors. Other causes include local or systematic infections, vascular or tumor processes of the orbita $[7,8]$.

A cavernous haemangioma has to the best of our knowledge until now not been described with the leading symptoms of retroorbital pain with visual deficits. Cavernous haemangiomas of the optic nerve are rare. The first relevant article was published in 1978 by Fermaglich [9]. In their review of the literature in 2006, Lehner et al. found 42 cases of vascular malformations involving the anterior optic pathways, of which 30 were cavernous haemangiomas [3]. Hempelmann et al. published in 2007 the first case of complete resection of such a lesion which presented with acute haemorrhage in the perimesencephalic cistern and in the white matter of the right frontal lobe [10]. The lesion because of its cystic components was initially assumed to be a craniopharyngioma with intratumoral haemorrhage. Liu and colleagues found in their review 65 cases of cavernous malformations of the optic pathway and hypothalamus [1]. Krings et al., reported in year 2001 of a patient with an aneurysm of the middle cerebral artery and acute intraparenchymal bleeding. The operation which was performed showed that the etiology of the bleeding was not the asymptomatic aneurysm but a cavernous haemangioma which was diagnosed later via MRI [11].

As in our case, the review of Lehner and colleagues revealed a rapid improvement of visual deficits after excision of the lesion in up to $80 \%$ of the patients who were operated [3]. Since remnants of the malformation can lead to re-growth and bleeding, it is advisable to completely remove it whenever possible [1]. Gross total resection is the gold standard of therapy of cavernous malformations. Although decompression without complete resection usually leads to visual improvement, the risk of bleeding is not being reduced [1].

In our case the decline of visual acuity was gradual thus permitting a thorough presurgical work-up. In cases of acute symptomatology or in the extreme chiasmal apoplexy, immediate surgery has to be performed to avoid permanent damage to the visual pathway $[3,12]$.

In such cases the commonly available modality is most likely the CT. In other cases with not such acute onset, MRI is the preferred diagnostic study [13]. Especially gradient echo-sequences (T2 weighted images) are sensitive in revealing small haemorrhages and calcium. MRI is especially sensitive in small lesions, which involve for example the cranial nerves [14]. In our case and since the lesion mimicked an aneurysm of the internal carotid artery an angiography was also performed. However, generally MRI is available and in cases where an aneurysm is not being suspected, the angiography provides no additional information except for rare cases of coexisting venous angioma [15].

\begin{tabular}{|c|c|c|c|}
\hline Authors & Number of cases & Surgical Outcome & Surgical procedure \\
\hline Liu et al. [1] & 65 & Improvement of visual acuity in $85 \%$ & Gross total resection in $60 \%$ \\
\hline Lehner et al. [3] & 1 & Improvement & Excision \\
\hline Hempelmann et al. [10] & 1 & Improvement & Excision \\
\hline Lavin et al. [16] & 1 & Improvement & Decompression \\
\hline Mohr et al. [17] & 1 & Improvement & Decompression, biopsy \\
\hline Hassler et al. [15] & 3 & 2 improved, 1 deteriorated & Excision \\
\hline Steinberg et al. [18] & 2 & Improvement & 1 biopsy, 1 laser resection \\
\hline Shibuya et al. [14] & 2 & Improvement & Excision \\
\hline Warner et al. [19] & 1 & Improvement & Excision \\
\hline Iwai et al. [20] & 1 & Improvement & Excision \\
\hline Elmaci et al. [21] & 1 & Improvement & Excision \\
\hline Paladino et al. [22] & 1 & Improvement & Excision \\
\hline Desmukh et al. [23] & 4 & Improvement & Excision \\
\hline Ramina et al. [24] & 1 & Improvement & Excision \\
\hline Son et al. [25] & 1 & Unchanged & Excision \\
\hline Arrue et al. [13] & 3 & Improvement & 1 conservative, 1 excision, 1 partial resection \\
\hline Newmann et al. [12] & 1 & Improvement & Excision \\
\hline
\end{tabular}

Table 1: The table shows other cases of cavernous hemangiomas in the optic pathway, the treatment used and the postoperative outcome of the visual deficit. Notably, there was a marked improvement of the visual acuity in all patients where a total resection was performed. 
We see justification in presenting this case in order to reemphasize the necessity of thorough diagnostic evaluation of patients presenting with orbital, periorbital or retro-orbital pain and visual deficits. Pathologies of the optic nerve should always be included as potential diagnosis. In our case a tumor or aneurysm in the proximity of the optic nerve was initially considered. After advanced diagnostic work up however, we attributed the rare leading symptom of retro-orbital pain to a prechiasmatic intra-optic cavernoma. Microsurgical excision of the lesion and decompression of the optic nerve resulted in immediate resolution of the pain. The visual deficits showed marked improvement from post-op day one continuing to almost total resolution in the next few weeks. These results correspond with the literature and show that the best treatment for these lesions is the operative excision with excellent results (Table 1 ).

\section{Conclusion}

Rapid clarification of visual symptoms and retro-orbital pain with MRI scans and total excision of pathologies which compress but not diffusely infiltrate the optic pathways, should always be considered, since the postoperative results are usually very satisfactory and improve the quality of life drastically.

\section{References}

1. Liu JK, Lu Y, Raslan AM, Gultekin SH, Delashaw JB (2010) Cavernous malformations of the optic pathway and hypothalamus: analysis of 65 cases in the literature. Neurosurg Focus 29: E17.

2. Kivelev J, Niemelä M, Hernesniemi J (2010) Outcome after microsurgery in 14 patients with spinal cavernomas and review of the literature. J Neurosurg Spine 13: $524-34$

3. Lehner M, Fellner FA, Wurm G (2006) Cavernous haemangiomas of the anterior visual pathways. Short review on occasion of an exceptional case. Acta Neurochir 148: 571-8.

4. Prat R, Galeano I (2008) Endoscopic resection of cavernoma of foramen of Monro in a patient with familial multiple cavernomatosis. Clin Neurol Neurosurg 110: 834-7.

5. Brazis PW, Lee AG, Stewart M, Capobianco D (2002) Clinical review: the differential diagnosis of pain in the quiet eye. Neurologist 8: 82-100.

6. Swartz NG, Beck RW, Savino PJ, Sergott RC, Bosley TM, et al. (1995) Pain in anterior ischemic optic neuropathy. J Neuroophthalmol 15: 9-10.

7. Goldberg RA, Rootman J (1990) Clinical characteristics of metastatic orbital tumors. Ophthal 97: 620-4.

8. Goldberg RA, Rootman J, Cline RA (1990) Tumors metastatic to the orbit: a changing picture. Surv Ophthalmol 35: 1-24.

9. Fermaglich J, Kattah J, Manz H (1978) Venous angioma of the optic chiasm. Ann Neurol 4: 470-1.

10. Hempelmann RG, Mater E, Schröder F, Schön R (2007) Complete resection of a cavernous haemangioma of the optic nerve, the chiasm, and the optic tract. Acta Neurochir 149: 699-703.

11. Krings T, Mayfrank L, Thron A (2001) Bleeding from a cavernous angioma mimicking rupture of a middle cerebral artery aneurysm. Neuroradiology 43: 985-9.

12. Newman H, Nevo M, Constantini S, Maimon S, Kesler A (2008) Chiasmal cavernoma: a rare cause of acute visual loss improved by prompt surgery. Pediatr Neurosurg 44: 414-7.

13. Arrué P, Thorn-Kany M, Vally P, Lacroix F, Delisle MB, et al. (1999) Cavernous hemangioma of the intracranial optic pathways: CT and MRI. J Comput Assist Tomogr 23: 357-61.

14. Shibuya M, Baskaya MK, Saito K, Suzuki Y, Ooka K, et al. (1995) Cavernous malformations of the optic chiasma. Acta Neurochir 136: 29-36.

15. Hassler W, Zentner J, Petersen D (1989) Cavernous angioma of the optic nerve, Case report. Surg Neurol 31: 444-7.

16. Lavin PJ, McCrary JA, Roessmann U, Ellenberger C (1984) Chiasmal apoplexy: hemorrhage from a cryptic vascular malformation in the optic chiasm. Neurology 34: 1007-11.

17. Mohr G, Hardy J, Gauvin P (1985) Chiasmal apoplexy due to ruptured cavernous hemangioma of the optic chiasm. Surg Neurol 24: 636-40.

18. Steinberg GK, Marks MP, Shuer LM, Sogg RL, Enzmann DR, et al. (1990) Occult vascular malformations of the optic chiasm: magnetic resonance imaging diagnosis and surgical laser resection. Neurosurgery 27: 466-70.

19. Warner JE, Rizzo JF, Brown EW, Ogilvy CS (1996) Recurrent chiasmal apoplexy due to cavernous malformation. J Neuroophthalmol 16: 99-106.

20. Iwai Y, Yamanaka K, Nakajima H, Miyaura T (1999) Cavernous angioma of the optic chiasm--case report. Neurol Med Chir 39: 617-20.

21. Elmaci I, Ates G, Kurtkaya O, Necmettin Pamir M (2000) Chiasmal cavernous malformation, A rare cause of acute visual loss. J Neurosurg Sci 44: 226-9.

22. Paladino J, Rotim K, Pirker N, Gluncić V, Jurić G et al. (2001) Minimally invasive treatment of cavernous angioma of the optic chiasm: case report. Minim Invasive Neurosurg. 44: 114-6.

23. Deshmukh VR, Albuquerque FC, Zabramski JM, Spetzler RF (2003) Surgical management of cavernous malformations involving the cranial nerves. Neurosurg 53: 352-7.

24. Ramina K, Ebner FH, Ernemann U, Tatagiba M (2013) Surgery of cavernous hemangioma of the optic nerve: case report and review. J Neurol Surg A Cent Eur Neurosurg 74: 265-70.

25. Son DW, Lee SW, Choi CH (2008) Cavernous malformation of the optic chiasm: case report. J Kor Neurosurg Soc 44: 88-90. 


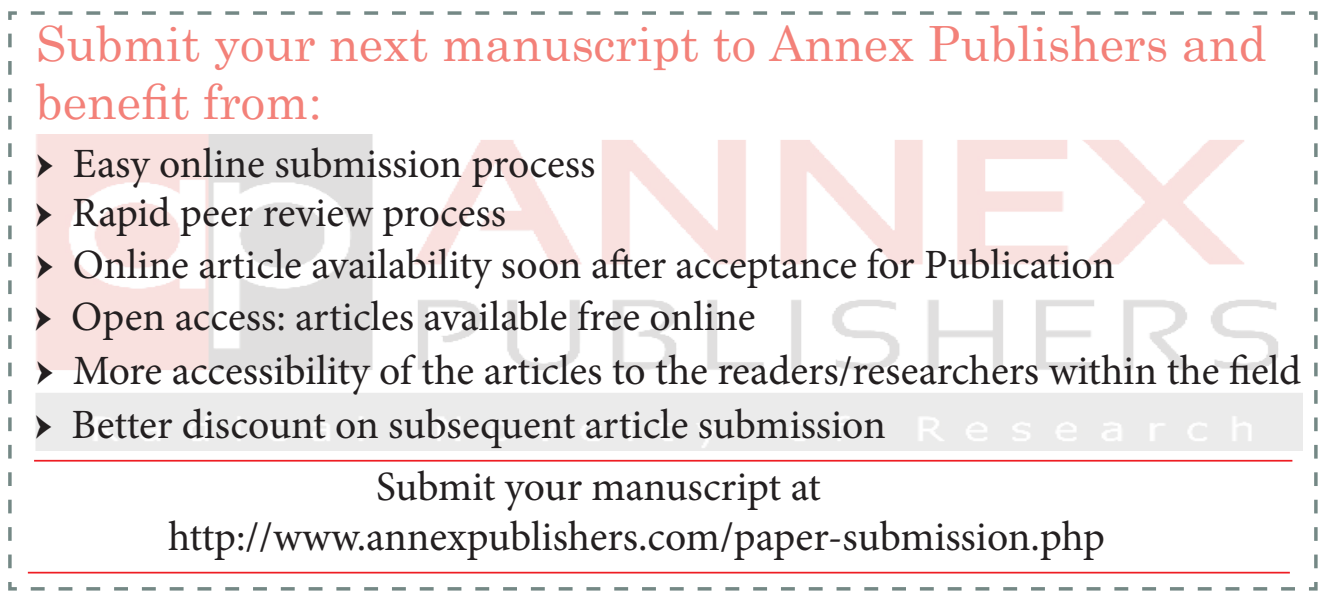

